\title{
The Importance of Evidence to Validate Clinical Practice and Its Significance in Making Recommendations
}

\author{
P. Marco Fisichella
}

Received: 10 July 2014 / Accepted: 11 July 2014 /Published online: 5 August 2014

(C) 2014 The Society for Surgery of the Alimentary Tract

We thank Drs. Cai and Liu for their interest in our paper. Clinical guidelines are considered the standards that guide clinical practice. Their importance in guiding patient management requires a very rigorous analysis and a painstaking scrutiny of the available evidence, which is carefully and laboriously selected, ranked, and analyzed objectively by panels of experts. We refer Drs. Cai and Liu to the American College of Cardiology/American Heart Association Task Force on Practice Guidelines to see how clinical practice guidelines are developed and the "science" behind them. $\left({ }^{1}\right)$ Therefore, until clinical practice guidelines on laparoscopic splenectomy for portal hypertension will be revised, we feel more comfortable following and referencing in our work the current ones rather than confiding on low-level evidence from isolated small case series, such as those referenced by Drs. Cai and Liu.

Drs. Cai and Liu comment that more evidence is required to validate the safety and feasibility of performing laparoscopic splenectomy for ITP patients with platelet count less than $10,000 / \mu \mathrm{L}$. We agree; we have merely shown that performing the operation in these conditions is feasible and safe in our hands, without generalizing our findings. $\left({ }^{2}\right)$
With regards to the use of the hand port, our data show that we could omit it most of the times. We appreciate the opinion of Drs. Cai and Liu, although they did fail to provide us and the readers with any evidence, or even their own data, to support their conclusions. Regrettably, Drs. Cai and Liu Until exhort to "recommendations" that have not been validated by a rigorous scientific process, like that used to establish guidelines.

Unfortunately, without data we are only left with opinions.

Conflicts of interest The author has no conflicts of interest to declare

\section{References}

1. Gibbons RJ, Smith S, Antman E; American College of Cardiology; American Heart Association. American College of Cardiology/ American Heart Association clinical practice guidelines: part I: where do they come from? Circulation. 2003;107(23):2979-2986

2. Fisichella PM, Wong YM, Pappas SG, Abood GJ: Laparoscopic Splenectomy: Perioperative Management, Surgical Technique, and Results. J Gastrointest Surg 2013

\section{P. M. Fisichella $(\bowtie)$}

Department of Surgery, Boston VA Healthcare System, Harvard Medical School, 1400 VFW Parkway. West Roxbury, 02132 Boston, MA, USA

e-mail: piero.fisichella@va.gov 